\title{
Myscéal 2.0: A Revised Experimental Interactive Lifelog Retrieval System for LSC'21
}

\author{
Ly-Duyen Tran* \\ Manh-Duy Nguyen* \\ Dublin City University \\ Ireland \\ Hyowon Lee \\ Insight Centre for Data Analytics, Dublin City University \\ Ireland
}

\begin{abstract}
Building an interactive retrieval system for lifelogging contains many challenges due to massive multi-modal personal data besides the requirement of accuracy and rapid response for such a tool. The Lifelog Search Challenge (LSC) is the international lifelog retrieval competition that inspires researchers to develop their systems to cope with the challenges and evaluates the effectiveness of their solutions. In this paper, we upgrade our previous Myscéal and present Myscéal 2.0 system for the LSC'21 with the improved features inspired by the novice users experiments. The experiments show that a novice user achieved more than half of the expert score on average. To mitigate the gap of them, some potential enhancements were identified and integrated to the enhanced version.
\end{abstract}

\section{CCS CONCEPTS}

- Information systems $\rightarrow$ Information retrieval; • Humancentered computing $\rightarrow$ Human computer interaction (HCI); User interface design.

\section{KEYWORDS}

lifelog, interactive retrieval system, elasticsearch

\section{ACM Reference Format:}

Ly-Duyen Tran, Manh-Duy Nguyen, Nguyen Thanh Binh, Hyowon Lee, and Cathal Gurrin. 2021. Myscéal 2.0: A Revised Experimental Interactive Lifelog Retrieval System for LSC'21. In Proceedings of the 4th Annual Lifelog Search Challenge (LSC '21), August 21, 2021, Taipei, Taiwan. ACM, New York, NY, USA, 6 pages. https://doi.org/10.1145/3463948.3469064

\section{INTRODUCTION}

The rapid development of low-cost wearable sensors has led to the emergence of lifelogging [14], which is the process of capturing personal digital evidence life traces. This personal digital data,

${ }^{*}$ Both authors contributed equally to this research.

Permission to make digital or hard copies of all or part of this work for personal or classroom use is granted without fee provided that copies are not made or distributed for profit or commercial advantage and that copies bear this notice and the full citation on the first page. Copyrights for components of this work owned by others than ACM must be honored. Abstracting with credit is permitted. To copy otherwise, or republish, to post on servers or to redistribute to lists, requires prior specific permission and/or a fee. Request permissions from Permissions@acm.org.

LSC '21, August 21, 2021, Taipei, Taiwan

(C) 2021 Association for Computing Machinery.

ACM ISBN 978-1-4503-8533-6/21/08 . \$15.00

https://doi.org/10.1145/3463948.3469064

\author{
Nguyen Thanh Binh \\ AISIA Research Lab \\ VNU HCM - University of Science \\ Vietnam \\ Cathal Gurrin \\ Dublin City University \\ Ireland
}

also known as lifelog, can contain images, location information, or biometric signals, dependent on which devices the person utilises. Although lifelogging has shown promise for many applications $[1,8,35]$, the fundamental challenge of searching through such massive archives in an effective manner is, as of yet, an unsolved task. There is a clear need for a highly usable interactive information retrieval tool for such archives.

Over the past five years, there have been a first generation of research challenges and associated datasets released for community use [11, 12, 29]. One such challenge is the Lifelog Search Challenge (LSC) workshop [13], which began in 2018. This annual challenge provides a large annotated lifelog dataset including photos, GPS location, and biometric information and supports a comprehensive evaluation of interactive lifelog retrieval systems in terms of speed and accuracy. Participating systems in the LSC need to facilitate a user to execute a descriptive information need, which is mentioned in a semantic query, and to locate an exact match from the large lifelog archive within a limited time period. Moreover, the ease of use of a system is an important challenge due to the requirement for systems to be used by novice users during the comparative evaluation.

According to the criteria described above, an efficient retrieval system has to process a query with a powerful search engine and provide the user with an intuitive user interface to facilitate efficient retrieval. The top performing system for the first LSC challenge in 2018 was built in the virtual environment [6] where users can fully operate easily while the top system in the second LSC [34] processed images as a sequence events of a video combining with its capacity of searching by drawing to optimize their seeking performance. The LSC challenge in 2020 introduced many new participating systems, one of which, Myscéal [37], incorporated a simple interface aimed at novice users and an efficient text search engine. This paper introduces a revised Myscéal 2.0 system that implements a number of improvements to make the retrieval system more competitive in 2021.

In order to improve the 2020 system, we carried out a novice user experiment to evaluate how Myscéal works for these newcomers. The experiment was configured the same way as the LSC challenge with similar evaluation metrics to have a fair comparison. By analyzing its result and the volunteers' feedback, we then introduce new features for Myscéal to present the enhanced version of it, named as Myscéal 2.0, to participate in the LSC'21 challenge. These extra features are not only about the internal search engine but 


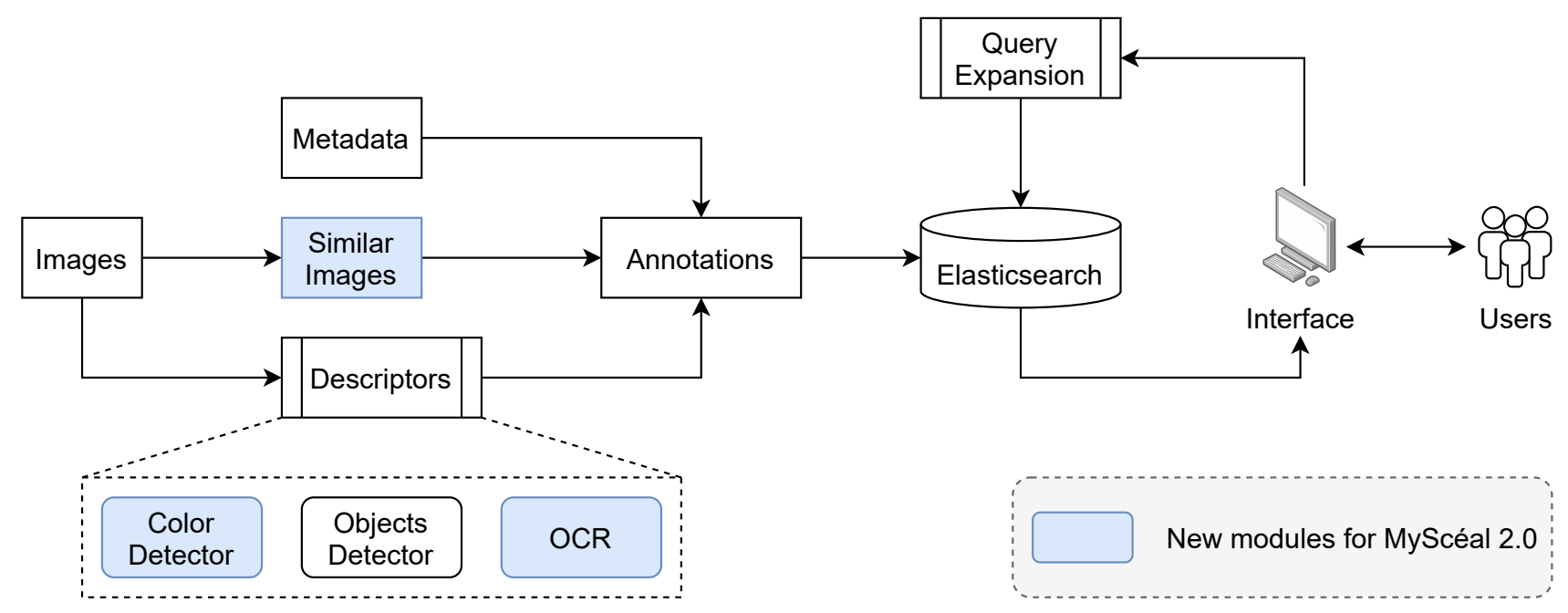

Figure 1: Myscéal 2.0 system pipeline follows its previous version with the extra module for color detector and the ability of text recognition (OCR). The visual similarity which inherits from the modified version of Myscéal participated in the ImageCLEFlifelog2020 challenge [38] also is integrated.

also the user interface (UI) of the system to make it become easier for users to interact with. Prior to that, we also briefly describe the previous system for readers to have deeper insights into how our system operates.

\section{RELATED WORK}

One of the earliest approaches for lifelog retrieving was to view the challenge as a form of database inquiry [9]. As the size of lifelog datasets increased, it became necessary to replace the database nonranked approach with a more conventional search-based approach that supports the provision of ranked output [14]. This idea was firstly applied in an official lifelog challenge by the LeMore [5] interactive system. In general such systems implement the concept of annotating each lifelog photo with semantic information of detected objects, location and activity context. This method allows a single image to be represented by a set of descriptive words, which supports text matching and ranked lists. Many lifelog retrieval tools in various lifelog benchmarking challenges were built on top of this standard but effective approach [16, 19, 21, 38], including all winners of the LSC challenges [6, 34, 37].

The most recent LSC competition (3rd in the series) had 14 participating teams, with a wide range of proposed algorithms to partake in the challenge. The liveXplore [21] and VIRET [18] are two systems that attended all LSC competitions to date. Both applications supported retrieval by implementing a drawing mechanism where users could sketch the general structure of an image need to be searched, and the result can be filtered by the textual annotation which is also the approach of the vitrivr system [16]. The DCU Voxento system [2] with its voice control feature counted the number of matching concepts between an inquiry and the semantic metadata of images as a ranking criterion. Similarly, the THUIR [22], Myscéal [37], and VRLE [7] system followed the document comparison idea in which the annotation of an image and a query are considered as textual documents. While the former team used the conventional
BM25 ranking function in the document retrieval field [32], the two latter systems applied a customised variation on the TF-IDF scoring function [37], which was based on regionalised object detection. Another common approach is to transform images metadata and queries into the same space through an embedding algorithm to be able to make the comparison. This idea was adopted by almost the half of the participants in the LSC'20 [17, 20, 26, 27, 39]. On the other hand, SOMHunter [27] which was the runner-up system of the competition and Exquisitor [17] heavily relied on user's relevance feedback, while LifeGraph [33] explored the potential of indexing the lifelog into a graph structure [30].

In this research, we enhance the top-performing system of the LSC' 20 challenge, Myscéal, and introduce the improved system called Myscéal 2.0 for LSC'21. The enhanced version integrates additional features and a refined user interface, in order to address some issues we found after conducting a novice user experiment independently of the LSC' 20 event. The experiments showed that although they were trained in a short period of time, novice users can utilise the essential features of the system without having considerable issues and obtain more than half of the score of the expert users. The novice users also suggest several ideas to cope with difficulties that they found during the experiments. The Myscéal 2.0 now has the capacity to recognize visible text in images as well as the dominant colors appearing in them. Some features from the modified version of Myscéal in the ImageCLEF lifelog challenge [38] are also adopted to enhance the performance of Myscéal 2.0. Our main contributions, therefore, are threefold. Firstly we set up a novice users experiment and report on it's findings. Secondly, we run an analysis based on the results to identify potential improvements to be made to Myscéal . Finally, we introduce new features and changes of the original system to overcome the issues and present the upgraded version Myscéal 2.0. 

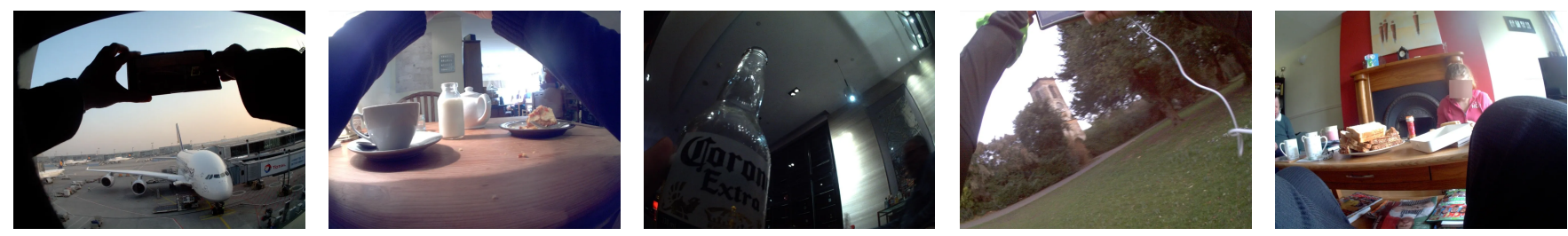

Figure 2: Solutions for 5 queries in our novice users experiments accordingly from left to right.

\section{THE LSC DATA AND METRICS}

The lifelog archive from the LSC'20 was reused for LSC'21 [15], though there were some images removed by the organisers. It was personal data gathered from four months during the years 2015 to 2018. The dataset contained data concerning the daily activities of a single lifelogger, who was the owner of the data and contained more than 190,000 images annotated with the visual concepts of detected objects appearing within them. This lifelog dataset also provided biometrics of the lifelogger, date, and GPS signals of where and when the photos were taken. All images are anonymized by blurring faces and sensitive information.

Regarding the evaluation metrics for the LSC, the accuracy and the retrieval time are both taken into account in calculating scores for each team [13]. Our Myscéal was developed to match with these metrics where its straightforward design of interface can help users to find the results promptly supported by the powerful search engine inside. The score for each correct retrieval, which is officially used in the LSC, is calculated by

$$
S=\max \left(0, P * \frac{M * 0.9^{i}-0.5 * t}{M}\right)
$$

which is influenced by both metrics. It is noted that $P$ and $M$ are the allowed maximum point and the maximum seeking time for each query accordingly. Since there is a huge gap in the experience between expert and novice users, $M$ can be configured specifically dependent on them. The $i$ indicates the number of incorrect submissions, and $t$ is denoted by the retrieval time of a user.

\section{THE MYSCÉAL SYSTEM}

\subsection{Myscéal System Operation}

In this section, we will briefly describe how Myscéal functioned for LSC'20, since it is the foundation for our LSC'21 system. However, readers are recommended to go through the original work to have a deeper understanding [37].

The pipeline of Myscéal is summarised in Figure 1. The system was inspired by a conventional lifelog retrieval engine [40] in which each image is stored in a database as a semantic form produced by a descriptor. The Myscéal , as depicted in Figure 1, used an object detector called DeepLabv3+ [4] to generate any object appearing within an image. This textual information was combined with the metadata, including the GPS location, date, activities, and visual concepts supported by the LSC organizer, then indexed in an opensource full-text search library Elasticsearch [10].

A user interacts with the system through a clean interface as illustrated in Figure 3 by entering a query in the search bar. The query was then enriched by a customised query expansion mechanism in which each term in the query would be mapped into specific words in the index based on its similarity scores. These scores were calculated by using Word2Vec model [28], and the synonyms, hypernyms, and hyponyms from WordNet [31]. These mapped terms were used to compute a ranked list of image surrogates using a novel scoring measurement called aTF-IDF [37], short for area term frequency-inverse document frequency, inspired from the original TF-IDF measure usually used in the information retrieval field. The idea behind it was similar to the TF-IDF, but the area (number of pixels) of a detected object was considered instead of the occurrence of its semantic data in the database.

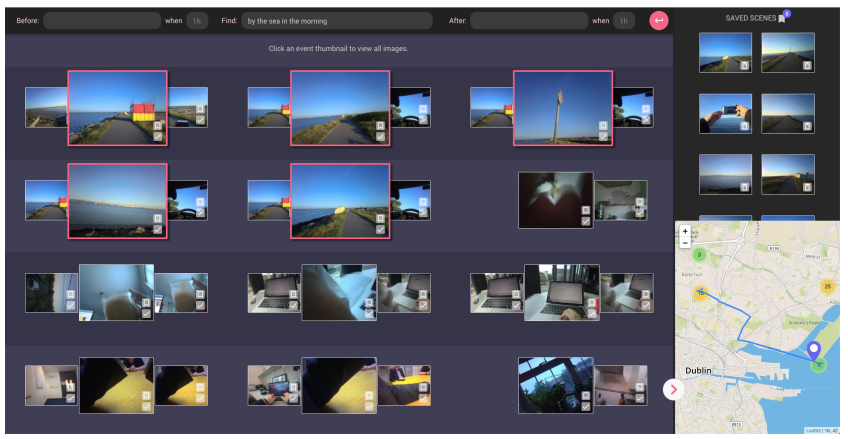

Figure 3: Myscéal user interface [37]

A common feature of many LSC systems was the provision of a faceted search panel for users to select filter items. In Figure 3, Myscéal did not have such a faceted filters panel. Instead, users could perform the filtering, for example, date or day-of-the-week, by inserting them into the search bar, which kept our interface clean and simple. Additionally, a map panel allowed a user to select a geographic reason for filtering results. Myscéal also introduced a temporal query mechanism to address a query that describes the information about the sequence of activities, which usually appears in the LSC challenge. This was facilitated by the provision of three separate query boxes (before, during, after) to support sequence specific information needs.

\subsection{Novice User Evaluation}

The essential idea of Myscéal was that it would be simple enough to be used by novice users. However, due to the Covid-19 pandemic, the LSC'20 did not facilitate a novice session, as it had done in previous years. The official sessions all required expert users, who were typically the system developers. Consequently, we conducted 
Table 1: Experiment score of 8 novice users compared to Myscéal team score in LSC'20

\begin{tabular}{|c|c|c|c|c|c|c|c|c|c|}
\hline Query & Myscéal & U1 & U2 & U3 & U4 & U5 & U6 & U7 & U8 \\
\hline 1 & 94.86 & 92.5 & 94.86 & 85 & 86.81 & 98.19 & 87.92 & 90.69 & 82.36 \\
2 & 78.06 & 54.86 & 50.69 & 68.61 & 47.08 & 59.03 & 57.92 & 43.33 & 59.58 \\
3 & 87.5 & 53.47 & 0 & 0 & 53.33 & 0 & 0 & 0 & 0 \\
4 & 78.61 & 0 & 77.5 & 51.11 & 0 & 52.5 & 0 & 64.44 & 0 \\
5 & 0 & 0 & 0 & 0 & 0 & 0 & 0 & 0 & 0 \\
\hline Total & 339.03 & 200.83 & 223.06 & 204.72 & 187.22 & 209.72 & 145.83 & 198.47 & 141.94 \\
\hline
\end{tabular}

our own novice user experiment to elicit feedback from novice users and evaluate system performance of Myscéal . A total of 8 volunteers participated in the experiment, and each of them had to solve 5 queries within a limited time period. All users used 3 sample queries in a pre-experiment training session. The experiment was then set up similarly to the LSC challenge, with each query bring incrementally refined with a hint every 60 seconds and have a total of 5 hints meaning that a user will have 6 minutes to find the solution. In all 5 official queries and 3 sample queries were selected from the query bank of the LSC'20 competition. They were ordered according to ascending level of difficulty and the 5 examining queries were:

- Q1: Taking a photograph of an A380 airplane in Germany before boarding a flight in the late afternoon in 2015 on the $19^{\text {th }}$ March.

- Q2: It was the best cake I had in years, in an antiques store. I was alone drinking tea and eating cake. I think I finished all the cake in 3 minutes. It was in the UK on a Saturday morning.

- Q3: I was having beer after a long day of meetings. It was a 'corona extra' beer in a bottle. I remember the room was dark. I was relaxing in a hotel lobby bar. I don't remember there was anyone else there. It was in May 2018, in Wuhan.

- Q4: Passing by a clocktower while running in a park near my home. It was in the early morning, around dawn. I drove to the park and I drove home again afterwards. It was a saturday morning in February.

- Q5: Four red figures, maybe they are aliens. It looked like a painting of aliens. There were walking on the desert. There was a big red wall behind the painting. And a TV, I think there was also a TV there. I was having tea and sandwiches in March 2015.

The score of each volunteer is shown in Table 1 where the Myscéal score is taken from the LSC'20 result generated by an expert user during the actual LSC'20 challenge. As depicted in Table 1 , all volunteers could not find the answer to the last query, including the expert. In general, the average total score of novice users was more than half of the author with $55.74 \%$. With 6 out of 8 , most novice users could solve three queries while the two remaining volunteers only completed two queries.

We observed that our map feature had shown its usefulness during the experiments when it played a critical role in the first four queries. Nevertheless, its position in the interface (bottom right corner) did not effectively grasp the attention of users. It suggests one minor change in our user interface. Furthermore, the fourth query also witnessed some novice users having difficulties identifying where the "home" location was on the map, although they had been trained about it. Therefore, the map would be better if there are some location annotations on it. The third and the fifth queries had shown a big disadvantage of Myscéal when it did not have text and color recognition. These features were visible in the solution images, as depicted in Figure 2. These two things are also the required features that we got from the feedback of novice users.

Additionally, they commented about our map filtering mechanism, which could be more prominent. We realized that our temporal retrieval feature had worked quite slowly and its visualization was still confusing for novice users. It was necessary to reorganize this feature to make it more easy-to-use for novice users. Another issue for consideration was that a novice user tended to fill in the search bar with every word that occurred in the query, leading to a noisy ranked list. This problem could be alleviated by our improved query expansion mechanism.

\subsection{Improvements for Myscéal 2.0}

Generally, the new version Myscéal 2.0 still uses the same pipeline as its original flow as illustrated in Figure 1 with the introduction of 3 new modules of visual similarity, color detection, and character recognition. We also make a few changes in the user interface as shown in Figure 4. Specifically, we consider the following upgrades for LSC'21.

Map position and visualization. To encourage the users to use the map utility more often, we decide to move the position of the map from the bottom-right of the screen into the top-right corner, which is next to the query section. We also increase the size of the map and thus make the saved section smaller. Some important locations, such as the lifelogger's home or workplace, are also noted on the map to help users better understand the data, reducing the seeking time. There are 421 different named places in the metadata of the whole dataset. However, we only choose a subset to visualize on the map, depending on the locations mentioned in the query.

Visual similarity and day summary. The system has been modified once to fit the Lifelog Moment Retrieval Task at ImageCLEFlifelog2020, where the visual similarity function and an overviewof-the-day row in the interface were introduced [38]. We brought these features into Myscéal 2.0. Regarding the former feature, the similarities between images are computed using the pretrained VGG16 model [36] combined with the visual local features [23-25]. This information can be seen as an annotation and is indexed in our database. A user can find similar images by clicking on a small visual similarity icon at the bottom of each photo as depicted in Figure 5. Figure 5 also presents the events view window, which is similar to Myscéal but with the addition of the last row for the 

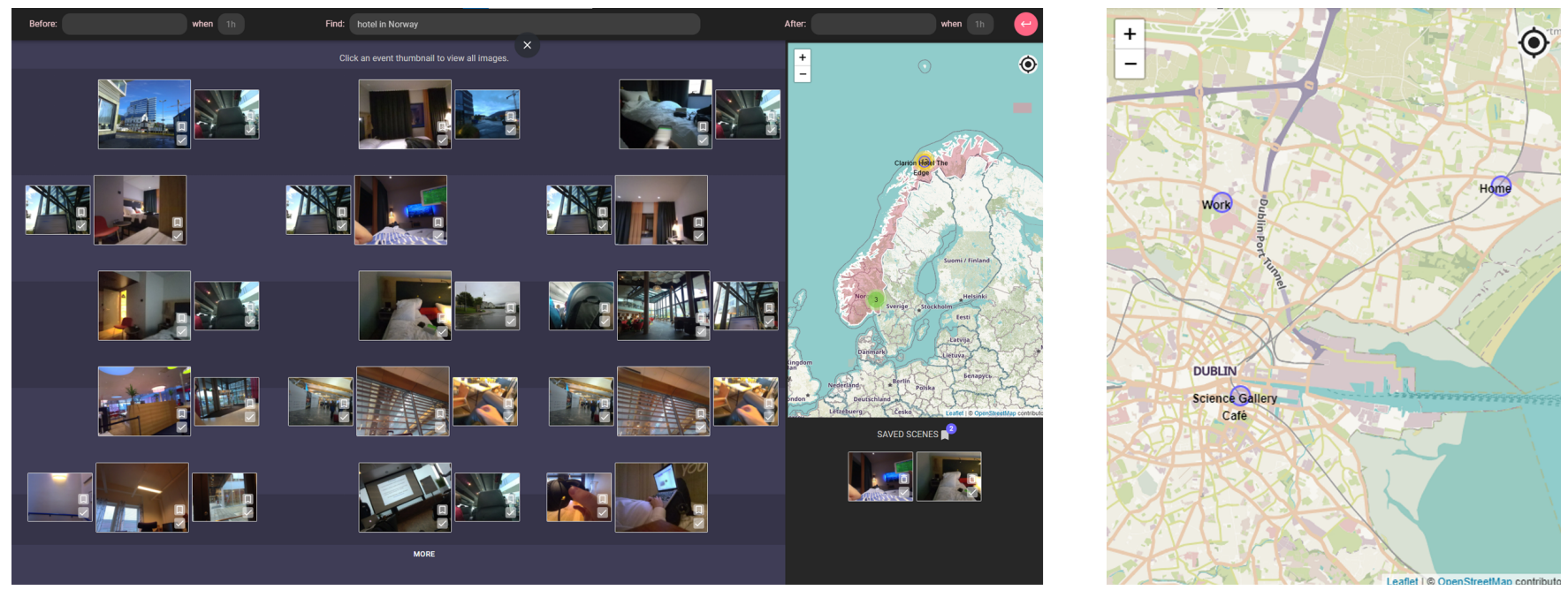

Figure 4: User interface of Myscéal 2.0 and the geographic map panel located at the top right corner of the UI.

day summarising feature. Each image in this row is a thumbnail of an activity that happens in the same location (walking in the airport, sitting inside the airplane). Using this, the user can browse the images through the day at a faster pace.

Additional information. We enrich the annotation associated with each image with color features and Optical Character Recognition information as well as Logo Detection for branded objects. The color features are generated by the pretrained bottom-up attention model [3] which can infer colors of detected objects in an image. Regarding the 2 remaining descriptors, we utilise the Microsoft Computer Vision API $^{1}$ service for the OCR and the logo recognition.

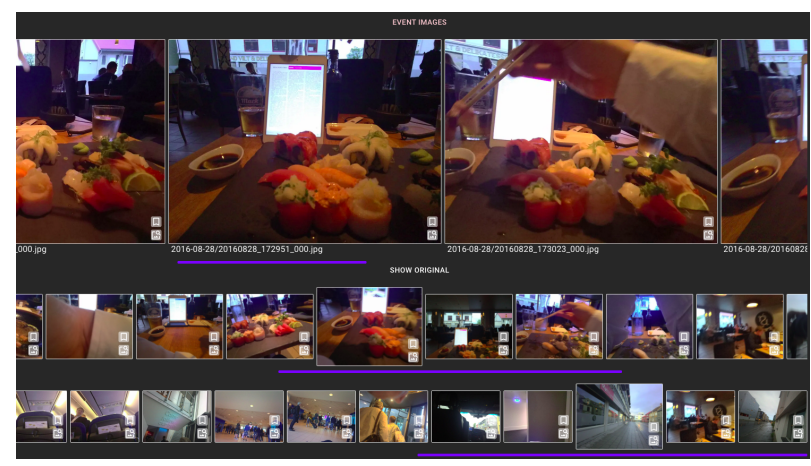

Figure 5: Events view window [38]

Improved query expansion. In the original version, each word in a query was expanded into a list of keywords in our indexed dictionary by the query expansion mechanism. For example, tea might imply the presence of a mug, or a teapot. However, novice users tend to enter most of the words in the given query leading to a noisy expansion output. In this version, we model the usefulness of the word from the user using similarity score and the frequency

\footnotetext{
${ }^{1}$ https://azure.microsoft.com/en-us/services/cognitive-services/computer-vision/
}

of each keyword in the dataset. If a word is considered not useful by the model, we notify the user by highlighting the word in the query and give the option to change or delete it to get a more accurate result with less noisy images.

Other utilities aiding novice users. A few utilities are also added to aid novice users in terms of saving time, including a reset button to clear the query and a zoomed view when the user hovers over the thumbnails. Moreover, a pop-up reminder is also used to remind the user to use a map whenever a location is mentioned in the query which is entered by the user.

\section{CONCLUSION}

In this paper, we describe a second generation interactive lifelog retrieval system developed for LSC'21. This system (Myscéal 2.0) was based in a first-generation retrieval system that ranked top of LSC'20. In order to enhance Myscéal 2.0, we report on a user experiment with novice users that took place after the LSC'20 workshop. The experiment revealed some weaknesses of our Myscéal system, including some important retrieval features that are missing as well as some usability bugs in presenting the interface options. Consequently we introduce an improved system with the extra modules of character and colour recognition with the re-design of the userinterface that more effectively supports and guides the searcher to suitable UI features. Two additional functions from the modified Myscéal in the ImageCLEFlifelog2020 [38] are also implemented in this enhanced version.

\section{ACKNOWLEDGMENTS}

This publication has emanated from research supported in party by research grants from Science Foundation Ireland under grant numbers SFI/12/RC/2289, SFI/13/RC/2106, 18/CRT/6223 and 18/CRT/6224.

\section{REFERENCES}

[1] Kiyoharu Aizawa, Yuto Maruyama, He Li, and Chamin Morikawa. 2013. Food balance estimation by using personal dietary tendencies in a multimedia food log. IEEE Transactions on multimedia 15, 8 (2013), 2176-2185. 
[2] Ahmed Alateeq, Mark Roantree, and Cathal Gurrin. 2020. Voxento: A Prototype Voice-Controlled Interactive Search Engine for Lifelogs. In Proceedings of the Third Annual Workshop on Lifelog Search Challenge (Dublin, Ireland) (LSC '20). Association for Computing Machinery, New York, NY, USA, 77-81.

[3] Peter Anderson, Xiaodong He, Chris Buehler, Damien Teney, Mark Johnson, Stephen Gould, and Lei Zhang. 2018. Bottom-up and top-down attention for image captioning and visual question answering. In Proceedings of the IEEE conference on computer vision and pattern recognition. 6077-6086.

[4] Liang-Chieh Chen, Yukun Zhu, George Papandreou, Florian Schroff, and Hartwig Adam. 2018. Encoder-Decoder with Atrous Separable Convolution for Semantic Image Segmentation. In ECCV.

[5] Gabriel de Oliveira Barra, Alejandro Cartas Ayala, Marc Bolaños, Mariella Dimiccoli, Xavier Giró Nieto, and Petia Radeva. 2016. Lemore: A lifelog engine for moments retrieval at the ntcir-lifelog lsat task. In Proceedings of the 12th NTCIR Conference on Evaluation of Information Access Technologies.

[6] Aaron Duane, Cathal Gurrin, and Wolfgang Huerst. 2018. Virtual reality lifelog explorer: lifelog search challenge at ACM ICMR 2018. In Proceedings of the 2018 ACM Workshop on The Lifelog Search Challenge. 20-23.

[7] Aaron Duane, Björn Pór Jónsson, and Cathal Gurrin. 2020. VRLE: Lifelog Interaction Prototype in Virtual Reality: Lifelog Search Challenge at ACM ICMR 2020 In Proceedings of the Third Annual Workshop on Lifelog Search Challenge (Dublin, Ireland) (LSC '20). Association for Computing Machinery, New York, NY, USA, $7-12$.

[8] Olga Gelonch, Mireia Ribera, Núria Codern-Bové, Sílvia Ramos, Maria Quintana, Gloria Chico, Noemí Cerulla, Paula Lafarga, Petia Radeva, and Maite Garolera. 2019. Acceptability of a lifelogging wearable camera in older adults with mild cognitive impairment: a mixed-method study. BMC geriatrics 19, 1 (2019), 110.

[9] Jim Gemmell, Gordon Bell, and Roger Lueder. 2002. MyLifeBits: fulfilling the Memex vision. Proceedings of the tenth ldots (2002), 235-238.

[10] Clinton Gormley and Zachary Tong. 2015. Elasticsearch: the definitive guide: a distributed real-time search and analytics engine. " O'Reilly Media, Inc."

[11] Cathal Gurrin, Hideo Joho, Frank Hopfgartner, Liting Zhou, and Rami Albatal. 2016. NTCIR Lifelog: The First Test Collection for Lifelog Research. Proceedings of the 39th International ACM SIGIR conference on Research and Development in Information Retrieval - SIGIR '16 (2016), 705-708. https://doi.org/10.1145/2911451. 2914680

[12] Cathal Gurrin, Hideo Joho, Frank Hopfgartner, Liting Zhou, Rashmi Gupta, Rami Albatal, Dang Nguyen, and Duc Tien. 2017. Overview of ntcir-13 lifelog-2 task. NTCIR.

[13] Cathal Gurrin, Klaus Schoeffmann, Hideo Joho, Andreas Leibetseder, Liting Zhou, Aaron Duane, Dang Nguyen, Duc Tien, Michael Riegler, Luca Piras, et al. 2019. Comparing approaches to interactive lifelog search at the lifelog search challenge (LSC2018). ITE Transactions on Media Technology and Applications 7, 2 (2019), 46-59.

[14] Cathal Gurrin, Alan F Smeaton, Aiden R Doherty, et al. 2014. Lifelogging: Personal big data. Foundations and Trends ${ }^{\circledR}$ in information retrieval 8, 1 (2014), 1-125.

[15] Cathal Gurrin, Björn Pór Jónsson, Klaus Schöffmann, Duc-Tien Dang-Nguyen, Jakub Lokoč, Minh-Triet Tran, Wolfgang Hürst, Luca Rossetto, and Graham Healy. 2021. Introduction to the Fourth Annual Lifelog Search Challenge, LSC'21. In Proc. International Conference on Multimedia Retrieval (ICMR'21). ACM, Taipei, Taiwan.

[16] Silvan Heller, Mahnaz Amiri Parian, Ralph Gasser, Loris Sauter, and Heiko Schuldt. 2020. Interactive Lifelog Retrieval with Vitrivr. In Proceedings of the Third Annua Workshop on Lifelog Search Challenge (Dublin, Ireland) (LSC '20). Association for Computing Machinery, New York, NY, USA, 1-6.

[17] Omar Shahbaz Khan, Mathias Dybkjær Larsen, Liam Alex Sonto Poulsen, Björn Pór Jónsson, Jan Zahálka, Stevan Rudinac, Dennis Koelma, and Marcel Worring. 2020. Exquisitor at the Lifelog Search Challenge 2020. In Proceedings of the Third Annual Workshop on Lifelog Search Challenge (Dublin, Ireland) (LSC '20). Association for Computing Machinery, New York, NY, USA, 19-22.

[18] Gregor Kovalčík, Vít Škrhak, Tomáš Souček, and Jakub Lokoč. 2020. VIRET Tool with Advanced Visual Browsing and Feedback. In Proceedings of the Third Annual Workshop on Lifelog Search Challenge (Dublin, Ireland) (LSC '20). Association for Computing Machinery, New York, NY, USA, 63-66.

[19] Nguyen-Khang Le, Dieu-Hien Nguyen, Vinh-Tiep Nguyen, and Minh-Triet Tran. 2019. Lifelog moment retrieval with advanced semantic extraction and flexible moment visualization for exploration. In CLEF2019 Working Notes. CEUR Workshop Proceedings, CEURWS. org< http://ceur-ws. org.

[20] Tu-Khiem Le, Van-Tu Ninh, Minh-Triet Tran, Thanh-An Nguyen, Hai-Dang Nguyen, Liting Zhou, Graham Healy, and Cathal Gurrin. 2020. LifeSeeker 2.0: Interactive Lifelog Search Engine at LSC 2020. In Proceedings of the Third Annual Workshop on Lifelog Search Challenge (Dublin, Ireland) (LSC '20). Association for
Computing Machinery, New York, NY, USA, 57-62.

[21] Andreas Leibetseder and Klaus Schoeffmann. 2020. LifeXplore at the Lifelog Search Challenge 2020. In Proceedings of the Third Annual Workshop on Lifelog Search Challenge (Dublin, Ireland) (LSC 20). Association for Computing Machinery, New York, NY, USA, 37-42.

[22] Jiayu Li, Min Zhang, Weizhi Ma, Yiqun Liu, and Shaoping Ma. 2020. A MultiLevel Interactive Lifelog Search Engine with User Feedback. In Proceedings of the Third Annual Workshop on Lifelog Search Challenge (Dublin, Ireland) (LSC '20). Association for Computing Machinery, New York, NY, USA, 29-35.

[23] D. G. Lowe. 1999. Object recognition from local scale-invariant features. In Proceedings of the Seventh IEEE International Conference on Computer Vision, Vol. 2. 1150-1157 vol.2.

[24] David G. Lowe. 2004. Distinctive Image Features from Scale-Invariant Keypoints. International fournal of Computer Vision 60, 2 (Nov 2004), 91-110. https://doi. org/10.1023/B:VISI.0000029664.99615.94

[25] H. Luo, H. Wei, and L. L. Lai. 2011. Creating Efficient Visual Codebook Ensembles for Object Categorization. IEEE Transactions on Systems, Man, and Cybernetics Part A: Systems and Humans 41, 2 (2011), 238-253.

[26] Anh-Vu Mai-Nguyen, Trong-Dat Phan, Anh-Khoa Vo, Van-Luon Tran, Minh-Son Dao, and Koji Zettsu. 2020. BIDAL-HCMUS@LSC2020: An Interactive Multimodal Lifelog Retrieval with Query-to-Sample Attention-Based Search Engine. In Proceedings of the Third Annual Workshop on Lifelog Search Challenge (Dublin, Ireland) (LSC '20). Association for Computing Machinery, New York, NY, USA, 43-49.

[27] František Mejzlík, Patrik Veselý, Miroslav Kratochvíl, Tomáš Souček, and Jakub Lokoč. 2020. SOMHunter for Lifelog Search. In Proceedings of the Third Annual Workshop on Lifelog Search Challenge (Dublin, Ireland) (LSC '20). Association for Computing Machinery, New York, NY, USA, 73-75.

[28] Tomas Mikolov, Ilya Sutskever, Kai Chen, Greg S Corrado, and Jeff Dean. 2013. Distributed representations of words and phrases and their compositionality. In Advances in neural information processing systems. 3111-3119.

[29] Dang Nguyen, Duc Tien, Michael Riegler, Liting Zhou, and Cathal Gurrin. 2018. Challenges and opportunities within personal life archives. (2018).

[30] Manh-Duy Nguyen, Binh T Nguyen, and Cathal Gurrin. 2021. Graph-Based Indexing and Retrieval of Lifelog Data. In International Conference on Multimedia Modeling. Springer, 256-267.

[31] Peter Oram. 2001. WordNet: An electronic lexical database. Christiane Fellbaum (Ed.). Cambridge, MA: MIT Press, 1998. Pp. 423. Applied Psycholinguistics 22, 1 (2001), 131-134. https://doi.org/10.1017/S0142716401221079

[32] Stephen Robertson and Hugo Zaragoza. 2009. The probabilistic relevance framework: BM25 and beyond. Now Publishers Inc.

[33] Luca Rossetto, Matthias Baumgartner, Narges Ashena, Florian Ruosch, Romana Pernischová, and Abraham Bernstein. 2020. LifeGraph: A Knowledge Graph for Lifelogs. In Proceedings of the Third Annual Workshop on Lifelog Search Challenge (Dublin, Ireland) (LSC '20). Association for Computing Machinery, New York, NY, USA, 13-17.

[34] Luca Rossetto, Ralph Gasser, Silvan Heller, Mahnaz Amiri Parian, and Heiko Schuldt. 2019. Retrieval of structured and unstructured data with vitrivr. In Proceedings of the ACM Workshop on Lifelog Search Challenge. 27-31.

[35] L. Signal, M. Smith, M. Barr, J. Stanley, T. Chambers, Jiang Zhou, A. Duane, G. Jenkin, A. Pearson, C. Gurrin, A. Smeaton, J. Hoek, and C. Ni Mhurchu. 2017. Kids'Cam: An Objective Methodology to Study the World in Which Children Live. American fournal of Preventive Medicine 533 (2017), e89-e95.

[36] Karen Simonyan and Andrew Zisserman. 2014. Very deep convolutional networks for large-scale image recognition. arXiv preprint arXiv:1409.1556 (2014).

[37] Ly-Duyen Tran, Manh-Duy Nguyen, Nguyen Thanh Binh, Hyowon Lee, and Cathal Gurrin. 2020. Myscéal: An Experimental Interactive Lifelog Retrieval System for LSC'20. In Proceedings of the Third Annual Workshop on Lifelog Search Challenge. 23-28.

[38] Ly-Duyen Tran, Manh-Duy Nguyen, Binh Thanh Nguyen, and Cathal Gurrin. 2020. An Experiment in Interactive Retrieval for the Lifelog Moment Retrieval Task at ImageCLEFlifelog2020. In CLEF2020 Working Notes (CEUR Workshop Proceedings). CEUR-WS.org < http://ceur-ws.org >, Thessaloniki, Greece. http: //ceur-ws.org/Vol-2696/paper_103.pdf

[39] Minh-Triet Tran, Thanh-An Nguyen, Quoc-Cuong Tran, Mai-Khiem Tran, Khanh Nguyen, Van-Tu Ninh, Tu-Khiem Le, Hoang-Phuc Trang-Trung, Hoang-Anh Le, Hai-Dang Nguyen, Trong-Le Do, Viet-Khoa Vo-Ho, and Cathal Gurrin. 2020. FIRST - Flexible Interactive Retrieval SysTem for Visual Lifelog Exploration at LSC 2020. In Proceedings of the Third Annual Workshop on Lifelog Search Challenge (Dublin, Ireland) (LSC '20). Association for Computing Machinery, New York, NY, USA, 67-72.

[40] Liting Zhou, Duc-Tien Dang-Nguyen, and Cathal Gurrin. 2017. A baseline search engine for personal life archives. In Proceedings of the 2 nd Workshop on Lifelogging Tools and Applications. 21-24. 